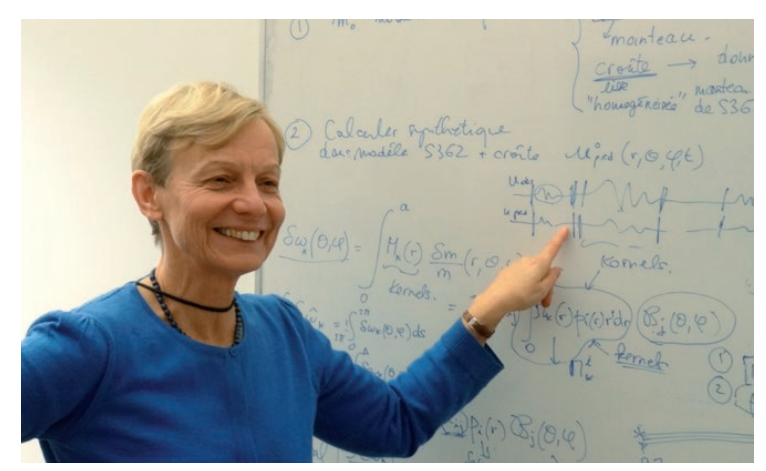

\title{
Barbara Romanowicz : une géophysicienne comblée
}

Interview réalisée par Véronique Pierron-Bohnes et Sandrine Morin, de la commission Femmes et Physique (F\&P) de la Société Française de Physique, le 3 décembre 2014 à l'Institut de Physique du Globe de Paris.

D'où vient votre vocation pour la recherche scientifique ? Le hasard... Il n'y avait pas de scientifique dans ma famille, ma mère était écrivain. Quand j'étais petite, j'étais passionnée par l'astronomie. Je faisais répéter à mes proches le nom et la taille des planètes. À l'école, j'étais surtout bonne en maths, même si j'aimais beaucoup les langues - j'étais bilingue français/polonais - et je me voyais bien dans l'interprétariat. Mais avec un 19 en maths au bac, j'ai intégré une classe préparatoire au lycée Saint-Louis, en mathématiques. Ensuite, je suis rentrée à l'École normale en maths, jusqu'en maîtrise (1974). Et là, tout à fait par hasard, dans les couloirs de l'université de Jussieu, j'ai vu une affiche pour un DEA (M2 actuel) en astronomie fondamentale qui proposait deux semaines de stage au Pic du Midi ! J'aime beaucoup la montagne et je me suis lancée!

Dans ce DEA d'astronomie, j'ai étudié l'effet des anomalies de masses à l'intérieur de la Terre sur les orbites des satellites artificiels. À l'époque (années 1970), tout le monde s'excitait plutôt sur la Lune, sur le fait qu'on y trouvait des points de forte densité : les mascons. J'ai fait ensuite une thèse de $3^{\mathrm{e}}$ cycle sur ces histoires de satellites en astronomie. Pendant cette thèse, j'ai rencontré des géophysiciens et ici à l'IPGP, un Australien, Kurt Lambeck, qui m'a proposé de faire une thèse d'État sur l'utilisation des données gravimétriques pour étudier l'intérieur de la Terre. À l'IPGP, j'ai côtoyé d'autres jeunes chercheurs qui faisaient de la sismologie. L'un d'entre eux, Georges Poupinet, étudiait les vitesses de propagation des ondes sismiques et en tirait des renseignements sur la structure interne élastique de la Terre : c'était le tout début de la tomographie sismique. Ce sujet m'a beaucoup plu. J'ai terminé ma thèse d'État en sismologie (1979) et, depuis, je suis restée dans ce domaine. Cela m'a permis d'associer mon goût des mathématiques et l'aspect pratique. Les deux me plaisent toujours aujourd'hui!

Vous avez toujours travaillé sur ordinateur ou vous avez aussi été sur le terrain ?

J'ai été un peu sur le terrain pendant ma thèse à l'IPGP. J'ai le souvenir d'une semaine dans les Pyrénées, passée à récupérer dans les stations sismiques les enregistrements qui, à l'époque, se faisaient sur papier. Après mon postdoc aux États-Unis, lors de la mise en place du réseau Géoscope, j'ai beaucoup voyagé. Ma présence sur le terrain avait pour but d'explorer les sites avant de les installer, avec de nombreuses discussions diplomatiques. À Berkeley, j’ai aussi participé à des visites de terrain sur des sites californiens très intéressants. Mais pour ma recherche au quotidien, j'ai besoin d'un ordinateur et de bases de données. Celles-ci, issues de travaux en réseaux, sont très importantes en sismologie. J'ai beaucoup œuvré au cours de ma carrière à la mise en place de ces réseaux sismiques.

Dans votre premier cours au Collège de France, vous racontez qu’Inge Lehmann, géophysicienne dans les années 1930, avait le sentiment qu'elle avait souvent perdu des compétitions en face d'hommes «bien moins compétents qu'elle ". L'avez-vous ressenti au cours de votre carrière ?

Elle avait eu le mot fameux « être en compétition avec des hommes incompétents ». Moi, je n'ai jamais eu ce sentiment, même si cela existe. Inge Lehmann a découvert la Graine, le noyau interne de la Terre, en 1936, à 54 ans. Les temps ont pas mal changé depuis. Pour ma part, j'estime avoir eu de la chance d'entrer à l'École normale au moment où les filles étaient séparées des garçons. C'était, je pense, de meilleures conditions de réussite pour les filles. Ma seule anecdote de compétition homme/femme date de mon séjour à Harvard, pendant mon doctorat : j'avais eu une meilleure note qu'un étudiant et il m'avait fait tout un discours comme quoi ce n'était pas juste parce que, lui, allait avoir la responsabilité d'une famille et qu'il fallait qu'il réussisse !

Lorsque vous avez été directrice d'un laboratoire, vous avez rencontré des problèmes de ce type ?

Non, pas du tout. J'ai plutôt pas mal réussi ma carrière et je ne me plains pas. Cependant, je ressens parfois que des résultats dans mon équipe ne sont pas mis en valeur aussi bien qu'ils auraient pu l'être, par manque de culot sans doute. Cet aspect-là a joué. Je suis consciente de ces problèmes parmi les étudiants. Les filles en général veulent vraiment être sûres de leur coup avant de présenter quelque chose, alors que les garçons présentent des résultats non encore confirmés plus facilement. Mais, finalement, ce n'est peut-être pas un désavantage !

Un des aspects difficiles pour beaucoup de femmes dans la recherche est le fait qu'elles doivent assumer la plus grosse partie des tâches familiales (enfant, logistique, parents), ce qui leur prend beaucoup de temps et les handicape pour leur carrière. Comment avez-vous concilié vie professionnelle et vie personnelle?

J'ai eu la chance d'avoir un mari qui a bien partagé les tâches, et aussi l'aide de ma famille. Le système français est par ailleurs plutôt favorable quand on a un poste au CNRS, avec la tranquillité d'esprit. 


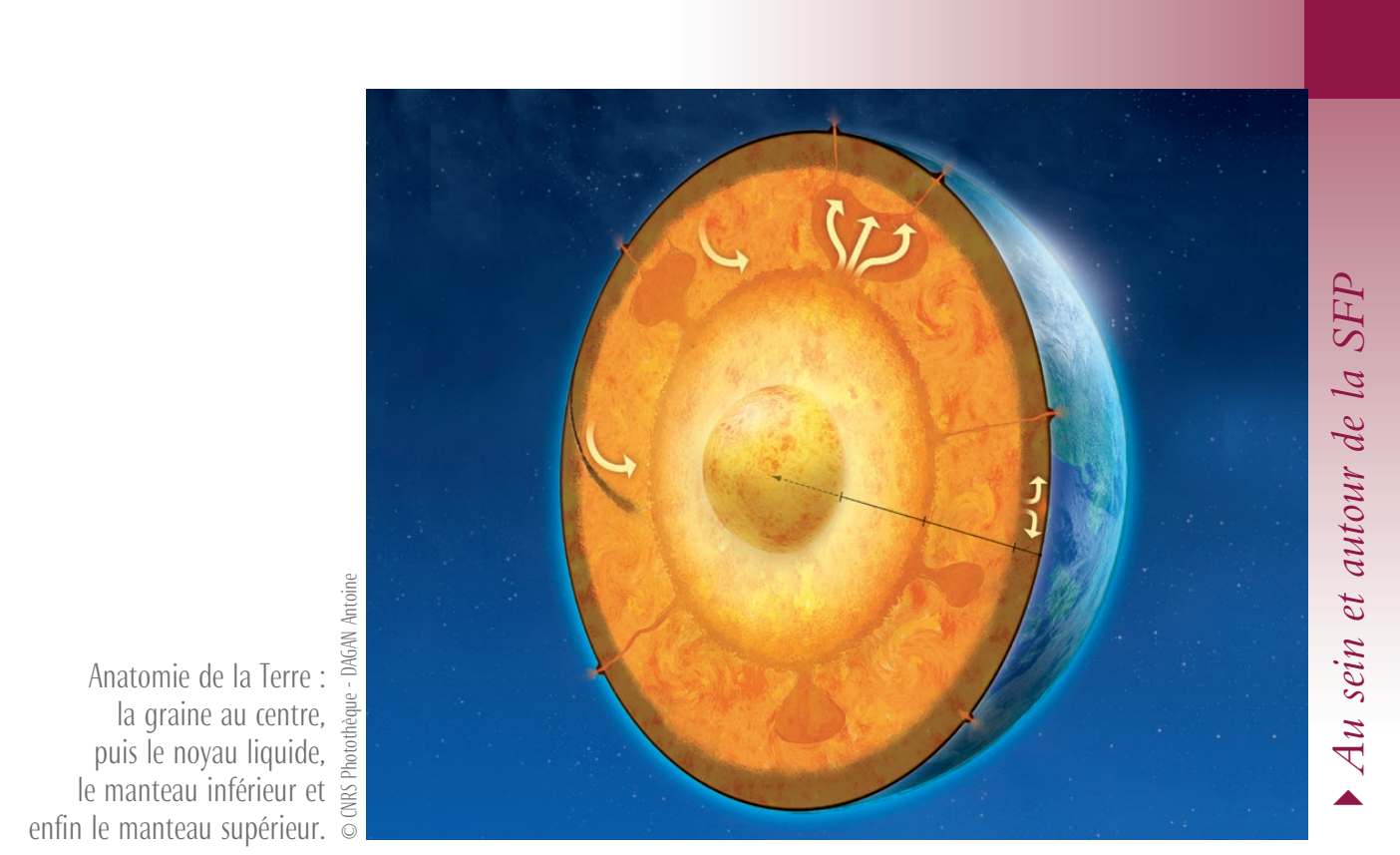

Vous avez effectué votre carrière entre Berkeley en Californie et Paris. Comment comparez-vous les modes de fonctionnement de la recherche dans les deux pays ?

J'ai beaucoup aimé les années à Berkeley, d'ailleurs j'y retourne souvent. Quand on m'a proposé le poste de professeure, j'ai trouvé que c'était une expérience très intéressante. Je n'avais pas forcément l'intention de rester aussi longtemps mais, du point de vue professionnel, aux États-Unis il y avait de nombreuses opportunités d'obtenir des crédits de recherche, alors qu'en France il n'y avait pas l'équivalent. Aux États-Unis, dans les années 90 tout était possible si on avait de bonnes idées. On avait 30 à $35 \%$ de chance d'obtenir un financement. C'était important. Aujourd'hui, c'est plutôt $10 \%$ (équivalent à l'ANR en France). J'ai aussi apprécié d'élever mes enfants là-bas. Ils ont fréquenté une école bilingue avec pas mal d'avantages. Les horaires étaient aussi plus sympathiques. Ils sont contents d'y être allés, mais le problème c'est que l'un a pris racine là-bas et que l'autre vit en Angleterre. Cela fait une famille écartelée. C'est aussi le risque !

Si vous regardez vos trente ans de géophysique, quelles ont été les grandes évolutions de votre recherche?

C'est une vaste question. Les dix premières années après mon post-doc aux États-Unis, cela a été le développement du réseau sismique. Ma recherche était alors plutôt théorique, en attendant des données de qualité. Par contre, depuis que je suis aux ÉtatsUnis, j'ai construit toute une série d'outils pour la tomographie sismique à grande échelle avec, à la fois, un volet méthodologique et un volet théorique. Ces modèles sont de plus en plus performants pour permettre de découvrir plus de détails dans les structures internes et mieux comprendre la dynamique. C'est le fil conducteur.

Quel est le but ultime de votre recherche?

C'est vraiment comprendre comment fonctionne la Terre, quelle est la machine qui provoque la tectonique des plaques, comment se fait le couplage entre la tectonique des plaques et les mouvements de convection dans le manteau sous-jacent.

Quelle sont pour vous les découvertes les plus formidables en géophysique pendant cette période ?

La découverte qui m'a le plus passionnée, c'est quand dans les années 98 on a mis en évidence la présence de modes propres de la Terre dans les enregistrements de bruit sismique. On a ensuite montré que cela venait du vent qui excite les vagues ordinaires sur les mers, vagues qui vont interagir entre elles pour former des

vagues à plus longue période qui elles-mêmes interagissent avec la Terre solide. Pendant un moment, les sismologues "longue période " ne parlaient plus que de cela !

Il y a eu aussi une autre révolution : la mise au point de méthodes de corrélation de bruit. Dans cette approche, on n'attend plus les séismes, mais on utilise le bruit de fond continu pour calculer les corrélations entre les enregistrements de paires de stations. L'équipe de Michel Campillo (Grenoble) a écrit les premiers articles exploitant ainsi le bruit de fond, et maintenant beaucoup d'études de la croûte sont faites par cette méthode. À courte période, ce bruit de fond vient aussi des océans : les vagues qui déferlent sur la plage engendrent des ondes de surface de la Terre solide. Cela donne un pic de bruit de 6 à 20 secondes de période, qui contient beaucoup d'énergie et est utile pour l'étude de la structure de la croûte, mais aussi depuis peu pour celle du manteau.

Si vous deviez donner un message aux jeunes qui sont en thèse ou en postdoc, qu'est-ce que vous leur diriez ?

Je leur dirai que tout le monde n'est pas fait pour la recherche. Il faut vraiment être passionné, et s'en rendre compte assez tôt car c'est quand même un métier difficile. On passe 90\% de son temps à faire des choses plutôt routinières, et on ne sait jamais quand arrivera la récompense. Donc, il ne faut pas être motivé par des résultats rapides, mais être patient et avoir confiance en soi. Si on est passionné, il faut persévérer et ne pas se laisser faire ; les bons arrivent toujours à trouver un poste, même en France aujourd'hui. Bien sûr ce n'est pas forcément facile, on n'arrive pas du premier coup, mais il ne faut pas forcément s'en inquiéter, il faut y croire.

\section{Parcours}

Née le 5 avril 1950 à Suresnes dans une famille polonaise, mathématicienne de formation, Barbara Romanowicz est une sismologue géophysicienne, spécialiste de la structure et de la dynamique de la Terre.

Chercheuse à I'Institut de physique du globe de Paris (IPGP), elle a dirigé Géoscope, un réseau sismologique français qui couvre le monde entier. Pendant vingt ans, elle a dirigé le laboratoire de sismologie de I'Université de Californie à Berkekey.

Depuis 2011, elle est professeure au Collège de France, titulaire de la Chaire de Physique de l'intérieur de la Terre, et professeure de géophysique à l'Université de Berkeley. Membre de l'Académie des sciences depuis 2013, elle a reçu de nombreuses distinctions comme la médaille d'argent du CNRS (1992), les médailles Alfred Wegener (1999) et Beno Gutenberg (2003) de I'Union européenne des géosciences, la médaille Inge Lehmann (2009) de l'Union américaine de géophysique. 